\title{
Quantum Brachistochrone Problem and the Geometry of the State Space in Pseudo-Hermitian Quantum Mechanics
}

\author{
Ali Mostafazadeh* \\ Department of Mathematics, Koç University, Sariyer 34450, Istanbul, Turkey
}

(Received 21 March 2007; published 26 September 2007)

\begin{abstract}
A non-Hermitian operator with a real spectrum and a complete set of eigenvectors may serve as the Hamiltonian operator for a unitary quantum system provided that one makes an appropriate choice for the defining the inner product of physical Hilbert state. We study the consequences of such a choice for the representation of states in terms of projection operators and the geometry of the state space. This allows for a careful treatment of the quantum Brachistochrone problem and shows that it is indeed impossible to achieve faster unitary evolutions using $\mathcal{P} \mathcal{T}$-symmetric or other non-Hermitian Hamiltonians than those given by Hermitian Hamiltonians.
\end{abstract}

PACS numbers: 03.67.Lx, 02.30.Yy, 03.65.Xp

Since the publication of the pioneering work of Bender and Boettcher [1] on non-Hermitian $\mathcal{P} \mathcal{T}$-symmetric Hamiltonian operators, there have appeared numerous research articles exploring the mathematical properties of such operators and their possible physical applications. Recently, it has been suggested that one can obtain arbitrarily fast quantum evolutions using a class of such Hamiltonians [2]. If true, this will have drastic consequences in quantum computation, because, for example, it removes the bound on the time-optimal unitary NOT operations [3] that is obtained within the framework of conventional (Hermitian) quantum mechanics [4-7]. As pointed out in [8], this seems to contradict the equivalence of the quantum theory based on such non-Hermitian Hamiltonians and the Hermitian quantum mechanics $[9,10]$. In this Letter, we offer a comprehensive treatment of this problem that is based on a detailed study of the projective Hilbert space $\mathcal{P} \mathcal{H}_{\text {phys }}$ of physical states. In particular, we obtain the explicit form of the natural metric tensor on $\mathcal{P} \mathcal{H}_{\text {phys }}$ and unravel the subtleties of the quantum Brachistochrone problem for a general unitary quantum system that is defined by a non-Hermitian Hamiltonian.

In general if a linear (possibly non-Hermitian) operator has a complete set of eigenvectors and a real spectrum, then it can serve as the Hamiltonian operator for a unitary quantum system provided that the physical Hilbert space of the system is defined using an appropriate inner product [11-13]. This leads to a quantum theory that turns out to be equivalent to the conventional quantum mechanics $[9,10]$. In other words, this theory, that we refer to as pseudoHermitian quantum mechanics [14], is an alternative representation of the conventional quantum mechanics. The key ingredient of this representation is that the inner product of the physical Hilbert space $\mathcal{H}_{\text {phys }}$ is determined by the Hamiltonian operator of the system. This has led to the discovery of an intriguing structural similarity between quantum mechanics and general theory of relativity [15]. It has also found applications in dealing with the Hilbertspace problem in quantum cosmology [16], the old prob- lem of constructing a unitary first-quantized quantum theory of Klein-Gordon fields [17], bound state scattering [18], and ghosts in certain quantum field theories [19].

In [2] the authors consider a class of two-level nonHermitian $\mathcal{P} \mathcal{T}$-symmetric Hamiltonians, define $\mathcal{H}_{\text {phys }}$ using the so-called $\mathcal{C P} \mathcal{T}$-inner product, and explore the evolution of state vectors, i.e., elements of $\mathcal{H}_{\text {phys }}$. They conclude that for a fixed initial and final state vectors, $\psi_{I}$ and $\psi_{F}$, one can obtain a Hamiltonian operator that evolves $\psi_{I}$ into $\psi_{F}$ in an arbitrarily short time $\tau$. In this Letter we reconsider this problem from the point of view that the true dynamics of physical states occurs in the projective Hilbert space $\mathcal{P} \mathcal{H}_{\text {phys }}$, i.e., the state space of rays in the physical Hilbert space; a proper treatment of the quantum Brachistochrone problem requires a closer look at $\mathcal{P} \mathcal{H}_{\text {phys }}$ and its geometry.

In Hermitian quantum mechanics, the Hilbert space $\mathcal{H}$ is defined by the usual $L^{2}$-inner product and the projective Hilbert space $\mathcal{P} \mathcal{H}$ is the projective space $\mathbb{C} P^{N-1}$, where $N(\leq \infty)$ is the dimension of $\mathcal{H}$. As pointed out in [7], the lower bound on the duration $\tau$ of the evolution is proportional to the geodesic distance (between the initial and final states) that is determined using the Fubini-Study metric tensor on $\mathbb{C} P^{N-1}$, [20]. This suggests constructing the analogous metric tensor on $\mathcal{P} \mathcal{H}_{\text {phys }}$. A convenient method of doing this is to represent the states (elements of $\mathcal{P} \mathcal{H}_{\text {phys }}$ ) by appropriate projection operators and use the relevant inner product on the space of linear (trace-class) operators acting in $\mathcal{H}_{\text {phys }}$ to induce the desired metric tensor on $\mathcal{P} \mathcal{H}_{\text {phys }}$.

Let $\mathcal{H}$ be a Hilbert space defined by the conventional $L^{2}$-inner product $\langle\cdot \mid \cdot\rangle$ and $H: \mathcal{H} \rightarrow \mathcal{H}$ be a linear diagonalizable operator [21] with a real discrete spectrum. Then there is a (positive-definite) inner product $\prec \cdot, \cdot \succ$ that renders $H$ self-adjoint, i.e., $\langle\psi, H \phi \succ=\prec H \psi, \phi \succ$ [11]. This inner product is not unique but has the general form $\prec \cdot, \cdot\rangle=\left\langle\cdot \mid \eta_{+} \cdot\right\rangle$ [23], where $\eta_{+}: \mathcal{H} \rightarrow \mathcal{H}$ is a positivedefinite operator satisfying the pseudo-Hermiticity condition: $H^{\dagger}=\eta_{+} H \eta_{+}^{-1},[13,24]$. Here and throughout this 
Letter we define the adjoint $A^{\dagger}$ of every linear operator $A$ using the $L^{2}$-inner product $\langle\cdot \mid \cdot\rangle$ through the condition $\langle\psi \mid A \phi\rangle=\left\langle A^{\dagger} \psi \mid \phi\right\rangle$. We say that $A$ is Hermitian if $A^{\dagger}=$ $A$. We also introduce the $\eta_{+}$-pseudo-adjoint $A^{\#}$ of $A$ that is defined by $A^{\#}:=\eta_{+}^{-1} A^{\dagger} \eta_{+}$[24]. This allows us to express the $\eta_{+}$-pseudo-Hermiticity of $A$, i.e., the condition $A^{\dagger}=$ $\eta_{+} A \eta_{+}^{-1}$, as $A^{\#}=A$.

The physical Hilbert space $\mathcal{H}_{\text {phys }}$ of the system whose dynamics is determined by the Hamiltonian operator $H$ is obtained by taking the linear span of the eigenvectors of $H$ in $\mathcal{H}$, endowing it with the inner product $\prec \cdot, \cdot \succ$ for some (metric operator) $\eta_{+}$, and completing the resultant inner product space. Clearly any linear operator $A$ acting in $\mathcal{H}_{\text {phys }}$ is self-adjoint if and only if $A^{\#}=A$. These operators constitute the physical observables of the system [14], the primary example being the Hamiltonian $H$.

The Hilbert space $\mathcal{H}_{\text {phys }}$ and the Hamiltonian $H$ define a pseudo-Hermitian quantum system that can be equivalently described by the standard Hilbert space $\mathcal{H}$ and the Hermitian Hamiltonian $h:=\eta_{+}^{1 / 2} H \eta_{+}^{-(1 / 2)}$, [9,10]. This means that there is a one-to-one correspondence between elements of $\mathcal{H}$ and $\mathcal{H}_{\text {phys }}, \psi \rightarrow \psi^{\prime}:=\eta_{+}^{1 / 2} \psi$, and the corresponding physical observables, $A \rightarrow A^{\prime}:=\eta_{+}^{1 / 2} A \eta_{+}^{-(1 / 2)}$, such that the expectation values coincide: $\frac{\langle\psi, A \psi\rangle}{\langle\psi, \psi\rangle}=$ $\frac{\left\langle\psi^{\prime} \mid A^{\prime} \psi^{\prime}\right\rangle}{\left\langle\psi^{\prime} \mid \psi^{\prime}\right\rangle}$.

We begin our investigation of the state space in pseudoHermitian quantum mechanics by identifying physical states with orthogonal projection operators onto the corresponding rays. Given a state vector $\psi \in \mathcal{H}_{\text {phys }}-\{0\}$, the corresponding orthogonal projection operator $\Lambda_{\psi}: \mathcal{H}_{\text {phys }} \rightarrow \mathcal{H}_{\text {phys }}$ is a self-adjoint operator $\left(\Lambda_{\psi}^{\#}=\right.$ $\Lambda_{\psi}$ ) satisfying $\Lambda_{\psi}^{2}=\Lambda_{\psi}, \Lambda_{\psi} \psi=\psi$, and $\Lambda_{\psi} \phi=0$ if $\prec \psi, \phi \succ=0$ [25]. These conditions imply

$$
\Lambda_{\psi}=\frac{|\psi\rangle\langle\psi| \eta_{+}}{\left\langle\psi \mid \eta_{+} \psi\right\rangle}=\frac{|\psi \succ \prec \psi|}{\prec \psi, \psi \succ},
$$

where $|\psi\rangle:=|\psi\rangle$ and $\prec \psi \mid:=\langle\psi| \eta_{+}[26]$.

The inner product $\prec \cdot, \cdot \succ$ of $\mathcal{H}_{\text {phys }}$ induces the following inner product on the space of (trace-class) operators acting in $\mathcal{H}_{\text {phys }}$ :

$$
(A, B):=\operatorname{Tr}\left(A^{\#} B\right)=\operatorname{Tr}\left(\eta_{+}^{-1} A^{\dagger} \eta_{+} B\right),
$$

where $\operatorname{Tr}(A):=\sum_{n} \prec \psi_{n}, A \psi_{n} \succ$ and $\left\{\psi_{n}\right\}$ is an arbitrary orthonormal basis of $\mathcal{H}_{\text {phys }}$, [27]. Using the definition of the inner product $\prec \cdot, \succ \succ$ and introducing $\psi_{n}^{\prime}:=\eta_{+}^{1 / 2} \psi_{n}$ which form an orthonormal basis in $\mathcal{H}$, we have $\operatorname{Tr}(A)=$ $\sum_{n}\left\langle\psi_{n} \mid \eta_{+} A \psi_{n}\right\rangle=\sum_{n}\left\langle\psi_{n}^{\prime} \mid \eta_{+}^{1 / 2} A \eta_{+}^{-(1 / 2)} \psi_{n}^{\prime}\right\rangle$. Here the last sum is the usual trace of $\eta_{+}^{1 / 2} A \eta_{+}^{-(1 / 2)}$, which, in view of the cyclic identity for the trace, coincides with the trace of $A$. This shows that " $T r$ " is identical with the conventional trace used in Hermitian quantum mechanics. We also note that (2) is the unique inner product that for a given orthonormal basis $\left\{\psi_{n}\right\}$ of $\mathcal{H}_{\text {phys }}$ renders $\left\{\Lambda_{\psi_{n}}\right\}$ orthonormal.
Next, we set $d \Lambda_{\psi}:=\Lambda_{\psi+d \psi}-\Lambda_{\psi}$ and define the metric on $\mathcal{P} \mathcal{H}_{\text {phys }}$ according to

$$
d s^{2}:=\operatorname{Tr}\left(d \Lambda_{\psi}^{\#} d \Lambda_{\psi}\right) .
$$

After miraculous cancellations of many terms in this lengthy calculation and using the identity $\operatorname{Tr}(|\psi\rangle \times$ $\langle\phi|)=\langle\phi \mid \psi\rangle$, we find

$$
\begin{aligned}
d s^{2} & =\frac{2\left[\prec \psi, \psi \succ \prec d \psi, d \psi \succ-|\prec \psi, d \psi \succ|^{2}\right]}{|\prec \psi, \psi \succ|^{2}} \\
& =\frac{2\left[\left\langle\psi \mid \eta_{+} \psi\right\rangle\left\langle d \psi \mid \eta_{+} d \psi\right\rangle-\left|\left\langle\psi \mid \eta_{+} d \psi\right\rangle\right|^{2}\right]}{\left|\left\langle\psi \mid \eta_{+} \psi\right\rangle\right|^{2}} .
\end{aligned}
$$

For the case that $\mathcal{H}$ is identified as $\mathbb{C}^{N}$ endowed with the standard Euclidean inner product, i.e., $\langle\psi \mid \tilde{\psi}\rangle:=\sum_{n=1}^{N} z_{i}^{*} \tilde{z}_{i}$, where $\psi=\left(z_{1}, z_{2}, \ldots, z_{N}\right)$ and $\tilde{\psi}=\left(\tilde{z}_{1}, \tilde{z}_{2}, \ldots, \tilde{z}_{N}\right)$, Eq. (4) takes the form

$$
d s^{2}=\sum_{i, j=1}^{N} g_{i j^{*}} d z_{i} d z_{j}^{*},
$$

where

$$
g_{i j^{*}}:=\frac{2 \sum_{p, q=1}^{N}\left[\eta_{p q} \eta_{j i}-\eta_{p i} \eta_{j q}\right] z_{p}^{*} z_{q}}{\left(\sum_{m, n=1}^{N} \eta_{m n} z_{m}^{*} z_{n}\right)^{2}}
$$

and $\left(\eta_{i j}\right)$ is the matrix representation of $\eta_{+}$in the standard basis of $\mathbb{C}^{N}$. If we identify $\eta_{+}$with the identity operator, (6) yields the Fubini-Study metric on $\mathbb{C} P^{N-1}$, [20]. To see this in more detail, consider the case $N=2$. Relabeling the entries of $\left(\eta_{i j}\right)$ in terms of the real parameters $a, b_{1}, b_{2}, c$ as

$$
\left(\eta_{i j}\right)=:\left(\begin{array}{cc}
a & b_{1}+i b_{2} \\
b_{1}-i b_{2} & c
\end{array}\right),
$$

using the homogeneous coordinate $\zeta:=z_{2} / z_{1}$ in the patch where $z_{1} \neq 0$, i.e., taking $\left(z_{1}, z_{2}\right) \rightarrow(1, \zeta) \rightarrow \zeta$ (which is equivalent to setting $z_{1}=1$ and $z_{2}=\zeta$ ), and letting $\zeta=$ : $x+i y$ with $x, y \in \mathbb{R}$ we obtain

$$
d s^{2}=\frac{2\left(d+4 b_{1} c x\right)\left(d x^{2}+d y^{2}\right)}{\left[a+2\left(b_{1} x-b_{2} y\right)+c\left(x^{2}+y^{2}\right)\right]^{2}},
$$

where $d:=a c-\left(b_{1}^{2}+b_{2}^{2}\right)=\operatorname{det}\left(\eta_{i j}\right)$. For $\eta_{i j}=\delta_{i j}$, i.e., $a=c=1$ and $b_{1}=b_{2}=0$, (8) reduces to the wellknown formula for the Fubini-Study metric on $\mathbb{C} P^{1}$ (which is a two-dimensional sphere of unit radius) [28].

Equations (1) and (4) show that because the inner product of the physical Hilbert space depends on the Hamiltonian, so do the orthogonal projection operators representing the states and the metric on the state space. This is the root of the subtleties of the Brachistochrone problem in pseudo-Hermitian quantum mechanics. There is a fundamental difference between this problem and its Hermitian analog. Its proper formulation as a standard variational problem must include fixed (Hamiltonian-independent) choices for the initial and final states (as opposed to unobservable state vectors) as well as for the geometry of the space in which one minimizes the travel time. One can apply the argument of [7] and use the results of [20] to 
identify the travel time with a multiple of the distance traveled by the evolving state in the state space $\mathcal{P} \mathcal{H}_{\text {phys }}$. This would make the lower bound on the travel time proportional to the geodesic distance between the initial and final states.

In the treatment of the problem offered in [2], both the boundary conditions and the very notion of distance depend on the choice of the Hamiltonian. Therefore, it is not clear whether the result corresponds to arbitrarily close initial and final states or arbitrarily short travel times for distant states. To conclude that one may achieve "faster than Hermitian quantum mechanics" evolutions, one must consider initial and final states with a fixed distance and consider whether one can find evolutions that take less time than the lower bound set by Hermitian quantum mechanics. In the remainder of this Letter we prove that this is indeed impossible [29];

Theorem. - The lower bound on the travel time (upper bound on the speed) of unitary evolutions is a universal quantity independent of whether the evolution is generated by a Hermitian or non-Hermitian Hamiltonian.

To prove this theorem consider an arbitrary nonHermitian Hamiltonian operator $H$ that generates a unitary time-evolution in the Hilbert space $\mathcal{H}_{\text {phys }}$ defined by a metric operator $\eta_{+}$. Let $\psi_{I}$ and $\psi_{F}=U(\tau) \psi_{I}$ be the initial and final state vectors, where $U(t):=e^{-(i t H / \hbar)}$ is the evolution operator for $H$ and $\tau \in \mathbb{R}^{+}$is the travel time. Then the evolution operator $u(t):=e^{-(i t h / \hbar)}$ for the Hermitian Hamiltonian $h:=\eta^{1 / 2} H \eta^{-(1 / 2)}$, which defines a dynamics in the Hilbert space $\mathcal{H}$, evolves $\psi_{I}^{\prime}:=\eta^{1 / 2} \psi_{I}$ into $\psi_{F}^{\prime}:=\eta^{1 / 2} \psi_{F}=u(\tau) \psi_{I}^{\prime}$ in time $\tau$. This follows from the fact that $U(t)$ is $\eta_{+}$-pseudo-unitary, i.e., $U(t)^{-1}=U(t)^{\#}=$ $\eta_{+}^{-1} U(t)^{\dagger} \eta_{+},[30]$. Next, recall that

$$
\eta_{+}^{1 / 2}: \mathcal{H}_{\text {phys }} \rightarrow \mathcal{H}
$$

is a unitary operator $[9,10]$, i.e., for all $\psi, \phi \in \mathcal{H}_{\text {phys }}$ $\prec \psi, \phi \succ=\left\langle\eta_{+}^{1 / 2} \psi \mid \eta_{+}^{1 / 2} \phi\right\rangle$. In view of this relation and (1), it is not difficult to show that while the state $\Lambda_{I}$ evolves into $\Lambda_{F}$ in $\mathcal{P} \mathcal{H}_{\text {phys }}$, the state $\Lambda_{I}^{\prime}:=\eta^{1 / 2} \Lambda_{I} \eta^{-(1 / 2)}$ evolves into $\Lambda_{F}^{\prime}:=\eta^{1 / 2} \Lambda_{F} \eta^{-(1 / 2)}$ in $\mathcal{P} \mathcal{H}$. Because the travel times are identical $(=\tau)$, the speed of the evolution generated by $H$ will be different from that generated by $h$ if and only if the length of the curve $\Lambda(t)$ joining $\Lambda_{I}$ to $\Lambda_{F}$ in $\mathcal{P} \mathcal{H}_{\text {phys }}$ is different from that of the curve $\Lambda^{\prime}(t)$ joining $\Lambda_{I}^{\prime}$ to $\Lambda_{F}^{\prime}$ in $\mathcal{P} \mathcal{H}$. The optimal-time evolution for $H$ corresponds to the case that $\Lambda^{\prime}(t)$ is a geodesic in $\mathcal{P} \mathcal{H}_{\text {phys. }}$. To prove the above theorem, therefore, it is sufficient to show that the geodesic distance between $\Lambda_{I}$ and $\Lambda_{F}$ in $\mathcal{P} \mathcal{H}_{\text {phys }}$ is identical with the geodesic distance between $\Lambda_{I}^{\prime}$ and $\Lambda_{F}^{\prime}$ in $\mathcal{P} \mathcal{H}$. This follows from the fact that the diffeomorphism $f: \mathcal{P} \mathcal{H}_{\text {phys }} \rightarrow \mathcal{P} \mathcal{H}$ that is induced by the unitary transformation (9), namely,

$$
f(\Lambda):=\eta^{1 / 2} \Lambda \eta^{-(1 / 2)},
$$

pulls back the Fubini-Study metric on $\mathcal{P} \mathcal{H}$ to the metric
(6) on $\mathcal{P} \mathcal{H}_{\text {phys }}$. In other words $f$ is an isometry. Probably the simplest way of showing this is to use (3) and (10), and the cyclic identity for the trace to establish

$$
d s^{2}=\operatorname{Tr}\left[f\left(d \Lambda_{\psi}\right)^{\dagger} f\left(d \Lambda_{\psi}\right)\right]
$$

and note that the Fubini-Study metric corresponds to $d s^{2}=$ $\operatorname{Tr}\left(d \Lambda_{\psi}^{\prime \dagger} d \Lambda_{\psi}^{\prime}\right)$ where $\Lambda_{\psi}^{\prime}:=\frac{|\psi\rangle\langle\psi|}{\langle\psi \mid \psi\rangle}$.

Because the distance between $\Lambda_{I}$ and $\Lambda_{F}$ in $\mathcal{P} \mathcal{H}_{\text {phys }}$ is the same as the distance between $\Lambda_{I}^{\prime}$ and $\Lambda_{F}^{\prime}$ in $\mathcal{P} \mathcal{H}$, and because given a non-Hermitian Hamiltonian $H$ that evolves $\Lambda_{I}$ into $\Lambda_{F}$ in time $\tau$ the Hermitian Hamiltonian $h$ evolves $\Lambda_{I}^{\prime}$ into $\Lambda_{F}^{\prime}$ in the same time $\tau$, the evolution speed for $H$ is identical to that of $h$. In particular, there is no advantage of using a non-Hermitian Hamiltonian $H$ as far as the lower bound on $\tau$ is concerned. This argument shows that a vanishing lower bound corresponds to arbitrarily close initial and final states; it can never be achieved for distant initial and final states. For the case of antipodal initial and final states [4], which is directly relevant to the problem of constructing unitary NOT operations in quantum computation [5], we can verify this statement directly.

First we note that without loss of generality we can confine our attention to two-level systems. If we represent the initial state $\Lambda_{I}$ by the state vector

$$
\psi_{I}=\left(\begin{array}{l}
1 \\
0
\end{array}\right)
$$

the antipodal (final) state $\Lambda_{F}$, that satisfies $\Lambda_{F} \Lambda_{I}=$ $\Lambda_{I} \Lambda_{F}=0$, will be represented by a state vector of the form

$$
\psi_{F}=\nu\left(\begin{array}{c}
\beta \\
-a
\end{array}\right)
$$

where $\nu$ is an arbitrary nonzero normalization constant, $\beta:=b_{1}+i b_{2}=\eta_{12}$, and we have enforced the condition $\left.\left\langle\psi_{F} \mid \eta_{+} \psi_{I}\right\rangle=\prec \psi_{F}, \psi_{I}\right\rangle=0$ and used (7). The initial and final states have the form

$$
\Lambda_{I}=\left(\begin{array}{cc}
1 & \frac{\beta}{a} \\
0 & 0
\end{array}\right), \quad \Lambda_{F}=\left(\begin{array}{cc}
0 & -\frac{\beta}{a} \\
0 & 1
\end{array}\right),
$$

respectively. This calculation shows that fixing the initial and final states puts a restriction on the choice of the metric operator $\eta_{+}$and consequently the allowed Hamiltonian operator $H$. One can show that for

$$
\psi_{F}=\left(\begin{array}{l}
0 \\
1
\end{array}\right) \text {, }
$$

the metric operator $\eta_{+}$must be diagonal and

$$
S_{z}=\frac{\hbar}{2}\left(\begin{array}{cc}
1 & 0 \\
0 & -1
\end{array}\right)
$$

satisfies $S_{z}^{\#}=S_{z}$, i.e., it is an observable. For general nondiagonal $\eta_{+}, S_{z}$ fails to be an observable; it does not describe the spin along the $z$ axis and its eigenstates are not the states of definite spin along the $z$ axis.

The general form of an $\eta_{+}$-pseudo-Hermitian two-level Hamiltonian for the most general $\eta_{+}$is given in [31]. To find the optimal-time evolution for the above boundary 
conditions, we can pursue three different approaches: (i) We can use the results of [31] to determine the general form of $H$ for a given value of $\beta / a$ and fix the remaining degrees of freedom in $H$ by minimizing the travel time; (ii) we can follow the approach of [7] and compute the minimum travel time by evaluating the geodesic distance between $\Lambda_{I}$ and $\Lambda_{F}$ using the metric (8); (iii) we can map the problem to the one in the standard Hilbert space $\mathcal{H}$ and use the known results for Hermitian Hamiltonians. Here we employ (iii), because it is more straightforward.

Let $\Lambda_{I}^{\prime}$ and $\Lambda_{F}^{\prime}$ be the corresponding states in $\mathcal{P} \mathcal{H}$. Then a quick calculation shows that $\Lambda_{I}^{\prime}$ and $\Lambda_{F}^{\prime}$ are also antipodal; $\Lambda_{I}^{\prime} \Lambda_{F}^{\prime}=\eta^{1 / 2} \Lambda_{I} \Lambda_{F} \eta^{-(1 / 2)}=0$. Therefore, as implied by the above theorem, the lower bound on the travel time $\tau$ is identical with $\hbar \pi /\left|E_{1}-E_{2}\right|$, where $E_{1}$ and $E_{2}$ are the eigenvalues of $h$, [7]. Note that because $h$ and $H$ are isospectral, $E_{1}$ and $E_{2}$ are also eigenvalues of $H$.

This calculation confirms the statement of the above theorem for the case that the initial and final states are antipodal states. It shows that for these boundary conditions the bound obtained for Hermitian Hamiltonians also applies for admissible non-Hermitian Hamiltonians. Therefore, non-Hermitian Hamiltonians (that are capable of generating unitary time-evolutions) do not offer any advantage in performing a faster unitary NOT-operation. This conclusion cannot be avoided unless one sacrifices unitarity. Note that the hypothetical setups that involve switching between Hermitian and non-Hermitian Hamiltonians at different times [2] would require a timedependent metric operator which in turn violates unitarity [32]. Therefore such scenarios cannot be used to undermine the general applicability of the above theorem.

In this Letter we examined the structure of the projective space $\mathcal{P} \mathcal{H}_{\text {phys }}$ of physical states in pseudo-Hermitian quantum mechanics. We derived the form of the natural metric tensor on $\mathcal{P} \mathcal{H}_{\text {phys }}$ and showed that as a Riemannian manifold it is isometric to the projective Hilbert space $\mathcal{P} \mathcal{H}$ of Hermitian quantum mechanics. Furthermore, we demonstrated that the time evolution in $\mathcal{P} \mathcal{H}_{\text {phys }}$ that is determined by a diagonalizable non-Hermitian Hamiltonian with a real spectrum has a mirror image in the usual state space $\mathcal{P} \mathcal{H}$ of Hermitian quantum mechanics. This is indeed a manifestation of the equivalence of pseudo-Hermitian (and in particular $\mathcal{P} \mathcal{T}$-symmetric) quantum mechanics with Hermitian quantum mechanics. A direct consequence of this equivalence is that physical quantities cannot differentiate between pseudo-Hermitian and Hermitian quantum mechanics. Pseudo-Hermitian quantum mechanics can only be useful as a technical tool particularly for dealing with systems with an infinite-dimensional Hilbert space where, unlike $H$, the equivalent Hermitian Hamiltonian $h$ is generically a complicated nonlocal operator. Typical examples are the imaginary cubic potential, $H=p^{2}+i x^{3}$, and the delta function potential with a complex coupling, $H=p^{2}+z \delta(x)$, where $z \in \mathbb{C}$, [33].

I wish to thank Zafer Gedik for bringing reference [5] to my attention. *amostafazadeh@ku.edu.tr

[1] C. M. Bender and S. Boettcher, Phys. Rev. Lett. 80, 5243 (1998).

[2] C. M. Bender, D. C. Brody, H. F. Jones, and B. K. Meister, Phys. Rev. Lett. 98, 040403 (2007).

[3] M. A. Nielson, M. R. Dowling, M. Gu, and A. C. Doherty, Phys. Rev. A 73, 062323 (2006).

[4] L. Vaidman, Am. J. Phys. 60, 182 (1992).

[5] N. Margolus and L. B. Levitin, Physica (Amsterdam) 120D, 188 (1998), and references therein.

[6] A. Carlini, A. Hosoya, T. Koike, and Y. Okudaira, Phys. Rev. Lett. 96, 060503 (2006).

[7] D. C. Brody and D. W. Hook, J. Phys. A 39, L167 (2006).

[8] D. Martin, arXiv:quant-ph/0701223.

[9] A. Mostafazadeh, J. Phys. A 36, 7081 (2003).

[10] A. Mostafazadeh, Czech. J. Phys. 54, 1125 (2004); quant$\mathrm{ph} / 0310164$.

[11] A. Mostafazadeh, J. Math. Phys. (N.Y.) 43, 2814 (2002); 43, 3944 (2002).

[12] C. M. Bender, D. C. Brody, and H.F. Jones, Phys. Rev. Lett. 89, 270401 (2002); Phys. Rev. Lett. 92, 119902 (2004).

[13] A. Mostafazadeh, J. Math. Phys. (N.Y.) 44, 974 (2003); See also A. Mostafazadeh, J. Phys. A 38, 3213 (2005).

[14] A. Mostafazadeh and A. Batal, J.Phys. A 37, 11645 (2004).

[15] A. Mostafazadeh, Phys. Lett. A 320, 375 (2004); J. Math. Phys. (N.Y.) 47, 072103 (2006).

[16] A. Mostafazadeh, Ann. Phys. (N.Y.) 309, 1 (2004).

[17] A. Mostafazadeh and F. Zamani, Ann. Phys. (N.Y.) 321, 2183 (2006).

[18] A. Matzkin, J. Phys. A 39, 10859 (2006).

[19] C. M. Bender, Rep. Prog. Phys. 70, 947 (2007).

[20] J. Anandan and Y. Aharonov, Phys. Rev. Lett. 65, 1697 (1990).

[21] This means that $H$ has a complete set of eigenvectors. For a mathematically more rigorous treatment see [22].

[22] A. Mostafazadeh, Czech. J. Phys. 56, 919 (2006).

[23] The $\mathcal{C P} \mathcal{T}$-inner products [12] correspond to a special class of the inner products $\prec \cdot, \cdot \succ,[13]$.

[24] A. Mostafazadeh, J. Math. Phys. (N.Y.) 43, 205 (2002).

[25] The self-adjointness and orthogonality of $\Lambda_{\psi}$ are dictated by the quantum measurement theory [22].

[26] Another way of deriving (1) is to use the Hermitian representation, i.e., demand that $\Lambda_{\psi}=\eta_{+}^{-(1 / 2)}\left(\frac{\left|\psi^{\prime}\right\rangle\left\langle\psi^{\prime}\right|}{\left\langle\psi^{\prime} \mid \psi^{\prime}\right\rangle}\right) \eta_{+}^{1 / 2}$.

[27] M. Reed and B. Simon, Functional Analysis (Academic, San Diego, 1980), Vol. I.

[28] T. Eguchi, P. B. Gilkey, and A. J. Hanson, Phys. Rep. 66, 213 (1980).

[29] This is a stronger result than showing the impossibility of arbitrarily short travel times for distant initial and final states. The latter may be simply established using the fact that the travel time is proportional to the geodesic distance defined by the metric (6).

[30] Z. Ahmed and S.R. Jain, Phys. Rev. E 67, 045106 (2003); A. Mostafazadeh, J. Math. Phys. (N.Y.) 45, 932 (2004).

[31] A. Mostafazadeh and S. Özçelik, Turk. J. Phys. 30, 437 (2006).

[32] A. Mostafazadeh, Phys. Lett. B 650, 208 (2007).

[33] A. Mostafazadeh, J. Phys. A 39, 10171 (2006); 39, 13495 (2006). 